\title{
Assessment of ventricular contractility and ventricular- arterial coupling with a model-based sensor
}

\author{
Thomas Desaive*, Bernard Lambermont*, Nathalie Janssen*, Alexandre Ghuysen*, \\ Philippe Kolh*, Philippe Morimont*, Pierre C. Dauby*, \\ Christina Starfinger**, Geoffrey M. Shaw***, J. Geoffrey Chase**
}

* Cardiovascular Research Center, University of Liege, Liege, Belgium (E-mail: tdesaive@ulg.ac.be)

**Department of Mechanical Engineering, University of Canterbury, Christchurch, New Zealand ***Department of Intensive Care Medicine, Christchurch Hospital, Christchurch, New Zealand

Corresponding Authors:

Dr T. Desaive, email: tdesaive@ulg.ac.be; tel : +32-4366-3733 


\begin{abstract}
:
Estimation of ventricular contractility and ventricular arterial coupling is clinically important in diagnosing and treating cardiac dysfunction in the critically ill. However, experimental assessment of indexes of ventricular contractility, such as the end-systolic pressure-volume relationship, requires a highly invasive manoeuvre and measurements that are not typical in an intensive care unit (ICU). This research describes the use of a previously validated cardiovascular system model and parameter identification process to evaluate the right ventricular arterial coupling in septic shock. Model-based ventricular arterial coupling is defined by the ratio of the end systolic right ventricular elastance $\left(E_{\text {esrvf }}\right)$ over the pulmonary artery elastance $\left(E_{p a}\right)$ or the mean pulmonary inflow resistance $\left(R_{p u l i n}\right)$. Results are compared to the clinical gold-standard assessment (conductance catheter method). Six anesthetized healthy pigs weighing $20-30 \mathrm{~kg}$ received a $0.5 \mathrm{mg} \mathrm{kg}^{-1}$ endotoxin infusion over a period of $30 \mathrm{~min}$ from T0 to T30, to induce septic shock and veno-venous hemofiltration was used from T60 onward. The results show good agreement with the gold-standard experimental assessment. In particular, the model-based right ventricular elastance $\left(E_{\text {esrvf }}\right)$ correlates well with the clinical gold standard $\left(R^{2}=0.69\right)$ and the model-based non-invasive coupling $\left(E_{\text {esrvf }} / R_{\text {pulin }}\right)$ follow the same trends and dynamics $\left(R^{2}=0.37\right)$. The overall results show the potential to develop a model-based sensor to monitor ventricular-arterial coupling in clinical real-time.
\end{abstract}

Keywords: Cardiovascular, Model, Intensive Care, Sensor, ventricular function, ICU 


\section{INTRODUCTION}

Blood flow creates a continuous interaction between the cardiac ventricle and the arterial tree. This interaction, known as ventriculo-arterial coupling, is the main determinant of stroke volume and ejection pressure because it relates effort or force capacity to output resistance or afterload. This concept, which can be applied to the systemic and pulmonary circulations, and was developed as a whole quantitative and structured theory by Sunagawa [1].

Theoretically, acute assessment of the ventriculo-arterial coupling should lead to optimal hemodynamic therapy [2]. The effect of the coupling is mainly seen in changes in ejection fraction and vascular resistances. However, these parameters do not completely characterize both ventricular contractility and cardiovascular energetics. Thus, it is clinically important to define an index reflecting the interaction between arterial and ventricular properties, to assess the adequacy of hemodynamic treatment.

However, the technical difficulties of instantaneous pressure and volume measurements requiring highfidelity, manometer-tipped catheters and ultrasonic microcrystals or conductance catheters have hampered routine clinical applications due to cost and invasiveness. In addition, it has been generally assumed that the frequency response of the fluid-filled pressure catheters that are commonly used would be insufficient for instantaneous measurements, but would be acceptable for mean pressure estimations. Equally, given these additional invasive measurements, assessment of this coupling requires an additional vena cava occlusion manoeuvre which carries added risk. Hence, the goal of regularly measuring ventriculo-arterial coupling remains elusive with existing measurement tools. 
Recently, mathematical models of the cardiovascular system (CVS) have been developed to assist medical professionals in differentiating and diagnosing CVS disturbances, such as pulmonary embolism [3], changes in positive end expiratory pressure (PEEP) $[4,5]$, the impact of inotrope therapy [6], and septic shock [7]. These CVS models are able to capture all the fundamental CVS pressure and volume values and trends when compared to measured experimental data. Importantly, they capture all clinically relevant trends that distinguish these disease states, hemodynamic disturbances and the impact of therapies. The goal is to use the models and readily available clinical measurements to enable noninvasive patient specific monitoring and diagnosis.

In particular, this study uses a CVS model and identification process $[3-5,7]$ to estimate right ventriculararterial coupling. This model-based approach offers a potentially reliable procedure to assess coupling without further invasive measurements or procedures. Equally importantly, it offers the potential to develop a model-based sensor to monitor this metric in clinical real-time turning readily available data into a clear, clinically relevant physiological picture. As such, it would offer significant benefit in assessing condition and guiding therapy. 


\section{MATERIALS AND METHODS}

All experimental procedures and protocols used in this investigation were reviewed and approved by the Ethics Committee of the Medical Faculty of the University of Liege. They were performed in accordance with the Guide for the Care and Use of Laboratory Animals as adopted and promulgated by the U.S. National Institutes of Health (NIH Publication No.85-23, revised 1996). The surgical preparation and experimental protocol are described in [8] with relevant details repeated here.

\subsection{Experimental protocol and physiological measurements}

Experiments were performed on 6 healthy pure pietran pigs of either sex weighing from 20 to $30 \mathrm{~kg}$. Data from 5 pigs was analysed and identified for this research because the measurements from one animal had to be omitted as they contained corrupted data. The animals were premedicated and anesthetized as described previously [8]. A micromanometer-tipped catheter (Sentron pressure-measuring catheter, Cordis, Miami, FL, U.S.A.) was inserted into the main pulmonary artery and a 14-mm diameter perivascular flow probe (Transonic Systems, Ithaca, NY, U.S.A.) was adjusted around the main pulmonary artery $2 \mathrm{~cm}$ downstream of the pulmonary valve. Left atrial pressure was measured with a micromanometer-tipped catheter and systemic blood pressure was monitored with a micromanometertipped catheter inserted into the descending thoracic aorta through the left femoral artery. A 7F, 12electrode (8-mm interelectrode distance) conductance micromanometer-tipped catheter (CD Leycom, Zoetermeer, The Netherlands) was inserted through the right ventricle (RV) infundibulum into the right ventricle and positioned so that all electrodes were in the RV cavity. A 6F Fogarty balloon catheter (Baxter Healthcare Corp., Oakland, Calif) was advanced into the inferior vena cava through a right femoral venotomy. Inflation of this balloon produced gradual preload reduction. 
After a 30 min stabilization period, the animals received a $0.5 \mathrm{mg} / \mathrm{kg}$ endotoxin infusion (Lipopolysaccharide from Escherichia Coli serotype 0127:B8; Sigma Chemical, St Louis, MO, USA) over a 30 min period (from T0 to T30). They underwent, from T60 onwards, a zero-balance continuous veno-venous hemofiltration $(\mathrm{CVVH})$ at a rate of $45 \mathrm{ml} / \mathrm{kg} / \mathrm{h}$. A $0.7 \mathrm{~m}^{2}$ large pore $(78 \AA)$ membrane with a cutoff of 80kDa (Sureflux FH 70, Nipro, Osaka, Japan) and a hemofiltration device Baxter BM 25-BM 14 (Baxter Health Care, Munich, Germany) were used. Ultrafiltrate was replaced in the postdilution mode by a bicarbonate-buffered hemofiltration fluid $\left(\mathrm{Na}^{+}: 150 \mathrm{mM}, \mathrm{K}^{+}: 3 \mathrm{mM}\right.$, bicarbonate: $\left.30 \mathrm{mM}\right)$ at a temperature of $37^{\circ} \mathrm{C}$.

Hemodynamic data included pulmonary artery pressure (PAP) wave, pulmonary blood flow wave, left atrial pressure, systemic arterial pressure, heart rate, RV pressure, RV volume and RV pressure-volume (PV) loops. These parameters were monitored online and recorded every 30 minutes from T0 to T240 during a short apneic phase and stored for subsequent analysis. All analog signals were sampled at 200 $\mathrm{Hz}$ and continuously converted to digital form with an appropriate system (Codas, DataQ Instruments Inc., Akron, OH, USA).

\subsection{Clinical assessment of ventricular properties}

Ventricular end-systolic elastance is the reference contractility parameter that characterizes ventricular systolic function. Clinically, it is defined by the slope of the ventricular end-systolic P-V relation (ESPVR). Experimentally, right ventricular PV loops were obtained using the conductance catheter method [9]. The RV end-systolic elastance $\left(E_{e s}^{E x p}\right)$ was then determined during a rapid inferior vena cava occlusion manoeuvre [10]. Preload was reduced by progressive inflation of the Fogarty balloon in the inferior vena cava for no more than 10s to avoid the intervention of a baroreflex mechanism. End-systolic PV relationship was determined by fitting a straight line through the end-systolic PV points. Due to its 
invasiveness and risk, the ethical considerations of this gold-standard clinical assessment preclude its regular clinical use.

\subsection{Clinical assessment of arterial properties}

Calculation of the arterial input impedance is accepted as being the most complete description of the hydraulic load faced by the ventricle [11]. This impedance is a complex number that can be estimated in the frequency domain using a windkessel model that is an electrical analogy of the vascular bed [12]. In this study, a four-element windkessel model (WK4) was used to analyze the flow conditions in the pulmonary circulation throughout the experimental process. In this model (Figure 1), a resistor $\left(\mathrm{R}_{2}\right)$ represents the resistive properties of the pulmonary vasculature. A capacitor (C) is placed parallel to $\mathrm{R}_{2}$ and describes the compliant properties of the pulmonary arterial tree. A second resistor $\left(\mathrm{R}_{1}\right)$ is placed at the input of the circuit and reflects the characteristic impedance of the proximal pulmonary stem. Finally, an inductance (L) is added in series to accounts for the inertial properties of the blood.

The windkessel model thus separates the calculation of every component of the input impedance (compliance, main and peripheral resistances) in the model. In the time domain, these elements can be combined to define an arterial elastance $\left(E_{a}^{E x p}\right)$, which reflects RV afterload [13]:

$E_{a}^{E x p}=\left(R_{1}+R_{2}\right) /\left[T_{s}+R_{2} C\left(1-e^{-T_{d} / R_{2} C}\right)\right]$

where $T_{s}$ and $T_{d}$ are respectively the systolic and diastolic intervals, while $R_{1}, R_{2}$ and $C$ can be estimated using a classical, well accepted procedure described previously [14]. 


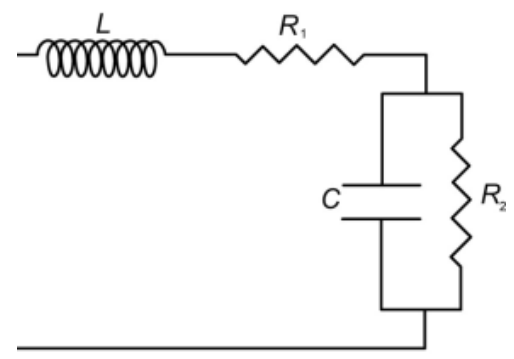

Fig. 1. The four-elements windkessel model (WK4). $\mathrm{R}_{1}=$ resistance of the main vessels, $\mathrm{R}_{2}=$ peripheral resistance, $\mathrm{C}=$ compliance and $\mathrm{L}=$ inductance.

\subsection{Ventriculo-arterial coupling}

The $E_{e s}^{E x p} / E_{a}^{E x p}$ ratio, obtained from P-V relations, characterizes the ventriculo-arterial interaction and is known as the ventriculo-arterial coupling index [15]. The efficiency of energy transfers between the ventricle and the arterial system strongly depends on this ratio. Under normal conditions, the right ventricle operates at a maximum efficiency and a submaximal stroke work $\left(E_{e s}^{E x p} / E_{a}^{E x p}>1\right)$, while uncoupling occurs when $E_{e s}^{E x p} / E_{a}^{E x p}<1[16-18]$.

\subsection{CVS Model}

The CVS model is a lumped parameter model of 6-8 elastic chambers with two active chambers for the left and right ventricles. The original model has been extended [3-7, 19, 20] and an overview of the 8 chamber version used in this research is given in Figure 2. Each chamber is characterized by the flow in and out of the chamber, the pressure up- and downstream, the resistances of the heart valves, and inertia of the blood. 


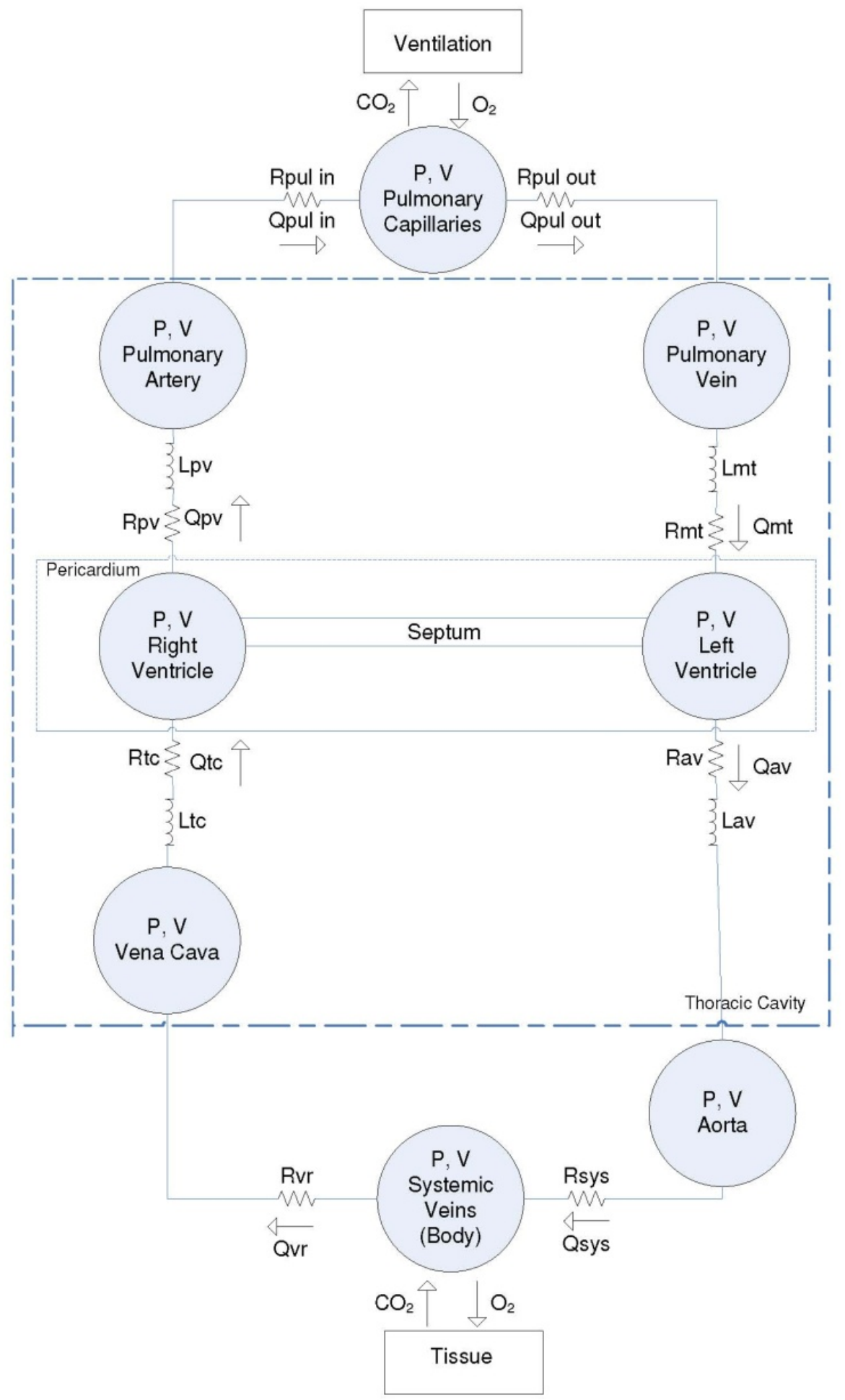

Fig. 2. Extended CVS model overview.

The CVS model uses the classical concept of time varying elastance proposed by Suga et al. [21] to simulate the cardiac muscle activation. More specifically, the upper and lower limits of the elastance are defined by the end-systolic pressure-volume relationship (ESPVR) and end-diastolic pressure--volume 
relationship (EDPVR) [22]. Combining ESPVR and EDPVR yields an equation relating the ventricular pressure $(\mathrm{P})$ to the ventricular volume $(\mathrm{V})$ :

$$
P=e(t) E_{e s r v f}\left(V-V_{d}\right)+(1-e(t)) P_{0}\left(e^{\lambda\left(V-V_{0}\right)}-1\right)
$$

where $\mathrm{P}_{0}, \mathrm{~V}_{0}$ and $\lambda$ are respectively, the pressure gradient and the volume at zero pressure and the curvature, while $\mathrm{E}_{\text {esrvf }}$ is the RV end-systolic elastance and $e(t)$ is the generic activation function (Figure 3) that accounts for ventricular activation $[19,20]$.

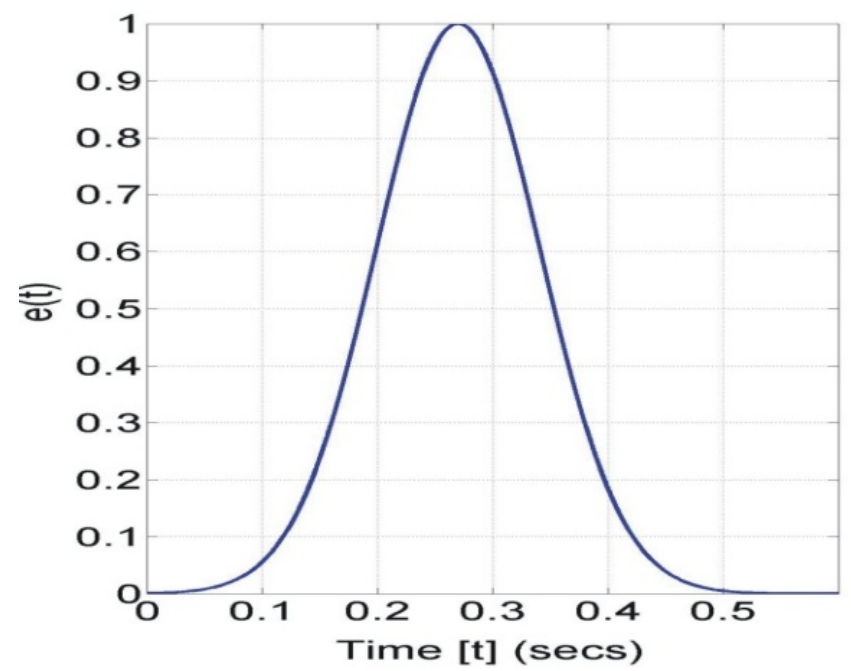

Fig. 3. Plot of the activation function e(t).

The pulmonary artery is also described by a simple passive pressure-volume relationship:

$$
P_{p a}=E_{p a} V_{p a}
$$

where $P_{p a}$ and $V_{p a}$ denote the pulmonary artery pressure and volume respectively. $E_{p a}$ is the arterial elastance. Using Equations 3 and 4, the identified right-ventricular coupling can be computed as the ratio $E_{\text {esrvf }} / E_{p a}$. 
A full list of the model equations and parameters is given in the appendix and further details can be found in references [3-7, 23-27].

\subsection{Model-based assessment of $E_{\text {esrvf }} / E_{p a}$ - Integral-based model identification}

The parameter identification method used in this research has already been shown to rapidly and accurately identify virtually the entire model parameter set in the presence of significant measurement noise and limited measurements [25]. It has been successfully tested on experimental animal models of pulmonary embolism [3], PEEP titrations and volume therapy decision support [4, 5], and septic shock experiments [7].

During the identification process only the systolic and diastolic values of both ventricular volumes, the pressures in the aorta $\left(P_{a o}\right)$ and in the pulmonary artery $\left(P_{p a}\right)$ are used. It is important to note that in this study, it was not intended to perfectly match the pressure and volume waveform shapes, but only the minimum (diastolic) and maximum (systolic) values. Hence, it seeks to capture the primary, clinically relevant dynamics for decision support at the bedside, rather than a potentially more perfect physiological picture.

In this model-based approach, the elastance defined in Equation $2\left(E_{\text {esrvf }}\right)$ is directly identified from the experimental data using the model and the identification method. The arterial compliance $\left(C_{p a}\right)$ and consequently the arterial elastance $\left(E_{p a}\right)$ in Equation 4 is evaluated from experimental data with the classical approximation $[28,29]$ 


$$
E_{p a}=1 / C_{p a}=\frac{P P_{a}}{S V}
$$

where $P P_{a}$ denotes the artery pulse pressure and $S V$ the stroke volume.

The arterial elastance from the windkessel model $\left(E_{a}^{E x p}\right)$ reflects the afterload. In the CVS model, two parameters may capture this effect. First, $E_{p a}$ defined in Equation 5. However, truer to the physiological situation, the mean pulmonary inflow resistance $\left(R_{\text {pulin }}\right)$ calculated during the model-based identification process, captures this effect as well [30,31]. More importantly, because it is identified directly from the data, it should better represent the afterload for each subject compared to the generic approximation in Equation 5.

Hence, this study examines two non-invasive model-based measures for the clinically assessed ventriculo-arterial coupling index $E_{e s}^{E x p} / E_{a}^{E x p}$, comparing both $E_{\text {esrvf }} / E_{p a}$ and $E_{\text {esrvf }} / R_{\text {pulin }}$. The goal is to obtain a non-invasive model-based measure that best captures the highly invasive and complex goldstandard clinical assessment, and is also physiologically representative and relevant.

The identification process is made every 30 minutes through the induced septic shock and hemofiltration. A value is identified at each point to assess these metrics. Thus, the ability of the model-based metrics to track immediate values and clinically expected trends are assessed. 


\section{RESULTS}

Figure 4 shows the mean (model-based) right ventricle end-systolic elastance $\left(E_{\text {esrvf }}\right)$ over all pigs during the experiment. This elastance is calculated during the model identification process and the value depends on the assumed volume at zero pressure $\left(V_{0}\right)$ for the ventricle used in the model. For reasons of simplicity, this value is assumed to be zero $\left(V_{0}=0 \mathrm{ml}\right)$ during the identification. However, $E_{\text {esrvf }}$ can easily be adjusted for a different, and potentially more realistic, $V_{0}$, as seen in Figure 4, where $E_{\text {esrvf }}$ is depicted for $V_{0}=0 \mathrm{ml}$ and $V_{0}=23 \mathrm{ml}$. These $V_{0}$ values span a realistic range [32].

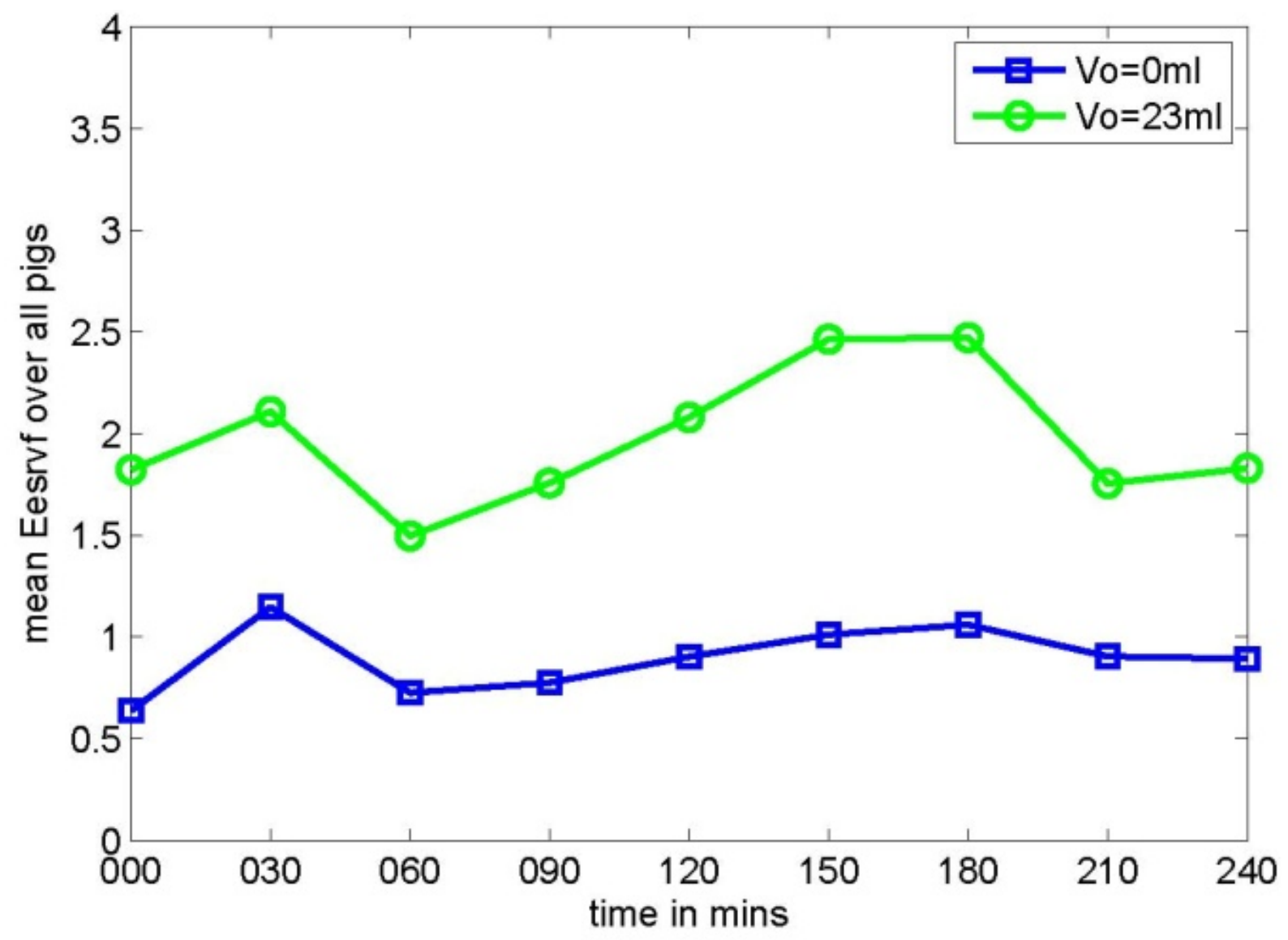

Fig. 4. Mean identified right ventricle elastance $\left(E_{\text {esrvf }}\right)$ for all 5 analysed pigs during the endotoxic shock experiment 
These identified $E_{\text {esrvf }}$ values (at $V_{0}=0 \mathrm{ml}$ ) are compared with previously reported clinically assessed values [8]. Figure 5 shows that the values and the resulting correlation trends match well $\left(R^{2}=0.69\right)$ with a trend line defined: $E_{e s}^{E x p}=0.22 * E_{\text {esrvf }}+0.45$. This line does not pass through the origin probably due to experimental errors on $E_{e s}^{E x p}$ and no pig-specific identification of $V_{0}$ from the data.

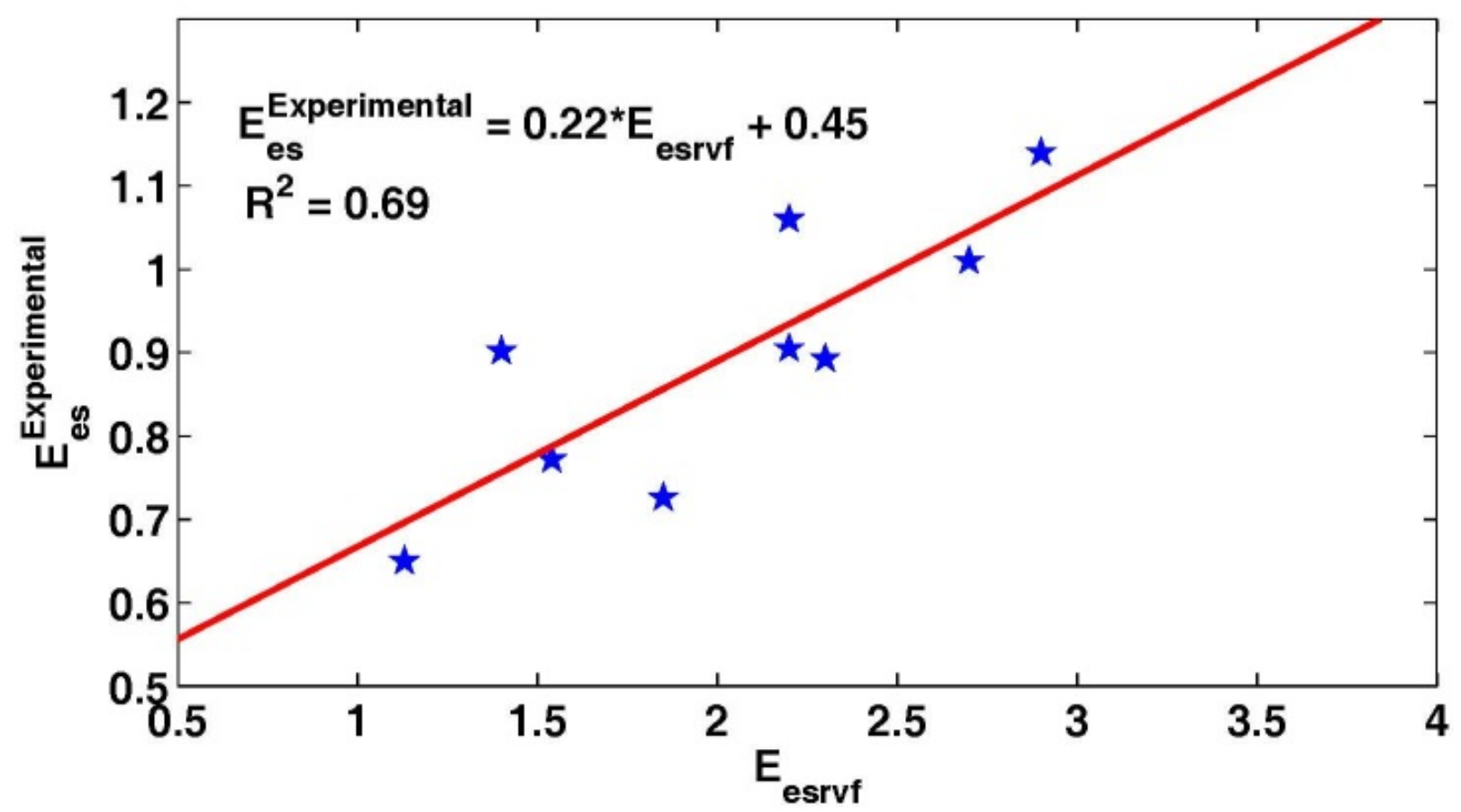

Fig. 5. Experimental vs simulated elastance for all 5 analysed pigs during the endotoxic shock experiment. The stars correspond to the mean measurements at each measurement time.

To compare the right ventricular-arterial coupling, we computed the first model identified coupling index $E_{\text {esrvf }} / E_{p a}$. Even if the simulated and experimentally derived elastances match accurately, as seen in Figure 5, a very poor correlation is found for the coupling ratio $\left(R^{2}=0.04\right)$.

This correlation is much better using $E_{\text {esrvf }} / R_{\text {pulin }}\left(R^{2}=0.37\right)$, as shown in Figure 6 . Figure 7 shows the better match of $R_{p u l i n}$ and $E_{a}^{E x p}$ that lead to these results. 


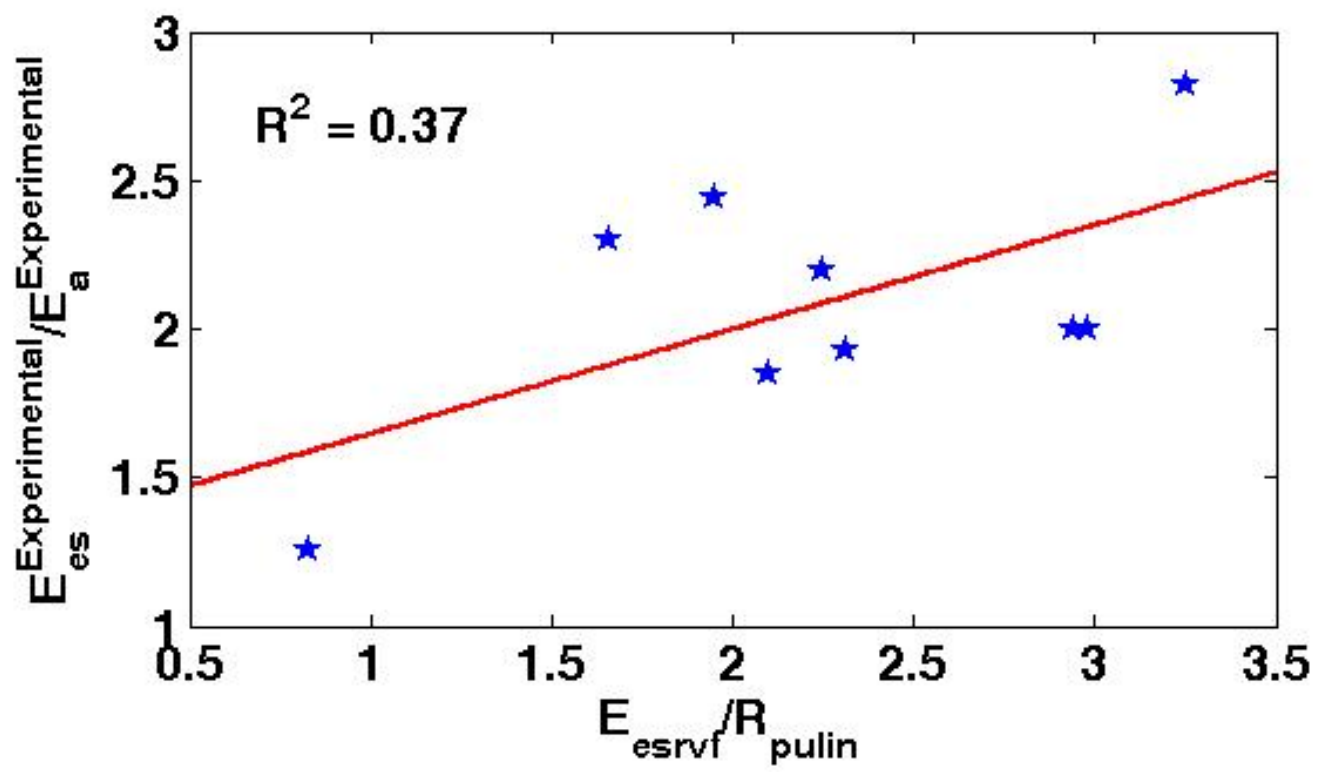

Fig. 6. Experimental coupling vs simulated coupling with $R_{\text {pulin }}$ for all 5 analysed pigs during the endotoxic shock experiment. The stars correspond to the mean measurements at each measurement time.

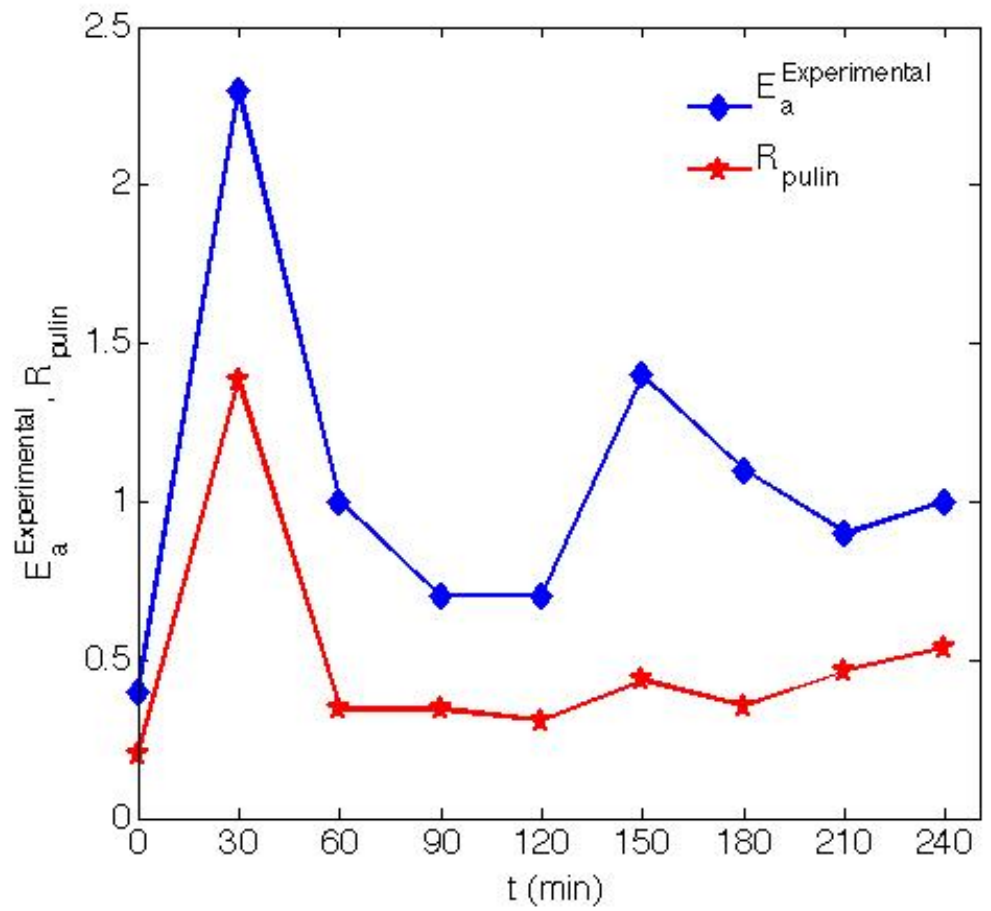

Fig. 7. $R_{p u l i n}$ and $E_{a}^{E x p}$ for all 5 analyzed pigs during the endotoxic shock experiment. 
Figure 8 presents the RV-vascular coupling using $E_{\text {esrvf }} / R_{\text {pulin }}$ during the endotoxic shock experiment as a further marker of the impact of sepsis. It shows the improved correlation between the new identified coupling metric and the experimental coupling. The overall trends over time, beginning with a sharp fall to a value approaching 1.0 and uncoupling, followed by a recovery due to hemofiltration treatment.

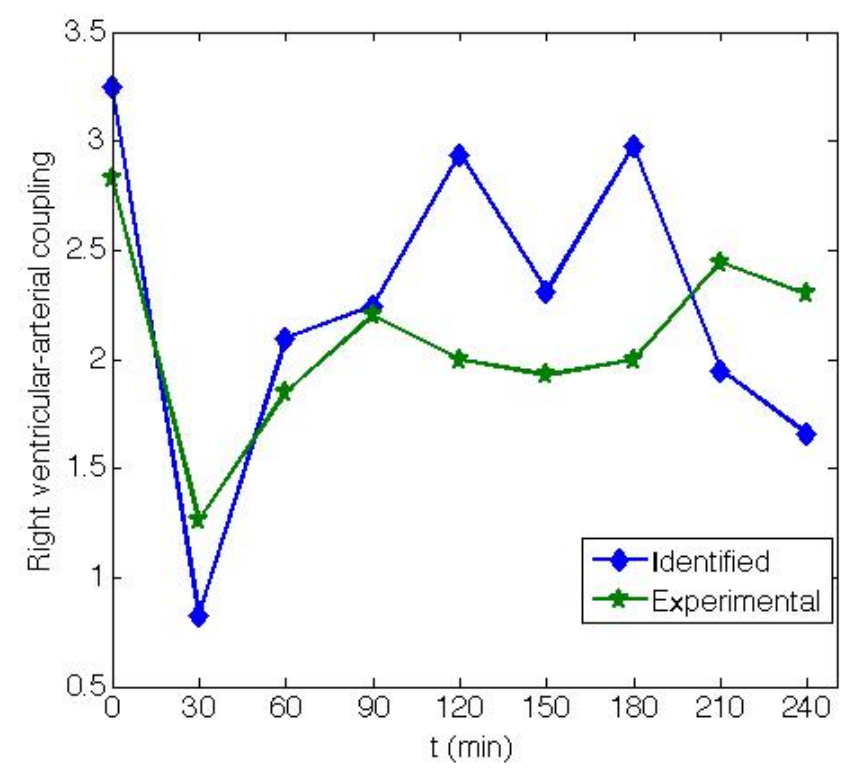

Figure 8: Mean experimental $\left(E_{e s}^{E x p} / E_{a}^{E x p}\right)$ and identified $\left(E_{\text {esrvf }} / R_{\text {pulin }}\right)$ right ventricular-arterial coupling as a function of time for all pigs. 


\section{DISCUSSION}

The major findings of this research are twofold. First, the clinical results obtained previously [8] are matched using a model-based metric that requires no invasive manoeuvres or added measurements. In Figure 8 it can be seen how hemofiltration, which starts at 60 min into the experiment, is able to prevent $\mathrm{RV}$-vascular uncoupling (ratio $<1.0$ ). This effect or behaviour is commonly observed during the late phases of endotoxic shock [33], and is accurately captured by the CVS model.

In particular, the data shows that in the early phases of endotoxic shock, RV-vascular coupling is preserved by an increase in RV contractility $\left(E_{\text {esrvf }}\right)$ as seen in Figure 4 . During the later stages and with CVVH, RV-vascular coupling is preserved where a decrease in pulmonary vascular resistance $\left(R_{\text {pulin }}\right)$, as seen in Figure 7, which is suggested to be the main reason for this result [8]. These results thus allow for a better understanding of the mechanisms of RV dysfunction during septic shock and thus have the potential to lead to more effective therapies and strategies for treating myocardial depression in septic shock. Equally, this clearer physiological picture is available from the model in clinical real-time.

Second, the results of this research present an important clinical outcome. They show that it is possible to relatively accurately calculate the right ventricular-arterial coupling without the need of an invasive preload reduction by doing this model-based approach. As mentioned previously, the assessment of RV contractility requires the occlusion of the vena cava, which raises obvious ethical and medical concerns and is far too difficult to perform as part of regular clinical practice. This model-based approach uses only a clinically realistic and minimal data set, and uses no data from these occlusion manoeuvres. Hence, the results presented are clinically accurate and realistic for a (potentially) typical CVS monitoring scenario without requiring any atypical measurements. 
The RV end-systolic elastance is directly identified from the model and the available experimental data. Using only minimum and maximum values of arterial pressure and ventricular volume, the identification process gives an accurate measure of the elastance.

Moreover, using the pulmonary resistance $\left(R_{\text {pulin }}\right)$ to characterize the RV afterload, there is no need to evaluate the arterial elastance using the parameters of the windkessel model, which has the main drawback of needing accurate pressure and flow waveforms [34] usually obtained via added manoeuvres and/or extensive signal conditioning. Equally, the windkessel model has its own limitation in representing an active, regulated non-linear system by a linear model. In contrast, the overall CVS model is non-linear and may better captures these effects.

Although a good correlation was found between the experimental and the model-based measures of the preload and the afterload, the resulting ratio assessing ventricular-arterial coupling using $E_{p a}$ shows a comparatively poor correlation of $R^{2}=0.04$. As the coupling ratio is defined by the ratio of the preload to the afterload, there can be a propagation of experimental errors. However, defining the RV ventriculararterial coupling with the model-based $R_{\text {pulin }}$

This outcome shows the importance of using subject-specific identified parameters instead of parameters estimated from the experimental data. Using $R_{p u l i n}$, a valid measure of afterload in the model context [25, 30, 31], also means that the entire ventriculo-arterial coupling index is defined by the models as identified directly from the data, without general or population assumptions. Hence, better consistency is enforced, which, in this case, results in a better result with this validated model.

Overall, this model-based approach thus offers potentially a new and more reliable procedure to assess the right ventricle contractility, as well as the right ventricular-arterial coupling. It also defines a new model-based metric $\left(E_{\text {esrvf }} / R_{\text {pulin }}\right)$ that, with adequate calibration, can effectively monitor coupling in 
clinical real-time and thus be used to guide therapy. In particular, because every element of this new metric is available at the bedside and can be assessed effectively continuously, it is a metric that can be used at any point in time the clinician wishes to assess, diagnose, and monitor patient condition, without added invasive measurements or manoeuvres.

\section{CONCLUSIONS}

This study shows the ability of a clinically validated CVS model to adequately and realistically capture the impact of pressure-volume changes during endotoxic shock and its treatment with CVVH. Comparable results to clinical experimental metrics that are not available at the (typical) ICU bedside for both values and clinical trends are obtained for the RV-vascular coupling. This research thus increases confidence in the clinical applicability and validity of this overall model-based diagnostic monitoring approach, and the underlying models and methods. 


\section{REFERENCES}

1. Sunagawa, K., K. Sagawa, and W.L. Maughan, Ventricular interaction with the loading system. Ann Biomed Eng, 1984. 12(2): p. 163-89.

2. Lambermont, B. and V. D'Orio, The role of right ventricular-pulmonary arterial coupling to differentiate between effects of inotropic agents in experimental right heart failure. Crit Care Med, 2006. 34(11): p. 2864-5.

3. Starfinger, C., et al., Model-based cardiac diagnosis of pulmonary embolism. Comput Methods Programs Biomed, 2007. 87(1): p. 46--60.

4. Starfinger, C., et al., Prediction of hemodynamic changes towards PEEP titrations at different volemic levels using a minimal cardiovascular model. Comput Methods Programs Biomed, 2008. 91(2): p. 128--134.

5. Starfinger, C., et al., Model-based identification of PEEP titrations during different volemic levels. Comput Methods Programs Biomed, 2008. 91(2): p. 135--144.

6. Chase, J.G., et al., Model-based prediction of the patient-specific response to adrenaline. The Open Medical Informatics Journal, 2010. 4: p. 149-163.

7. Starfinger, C., et al., Model-based identification and diagnosis of a porcine model of induced endotoxic shock with hemofiltration. Math Biosci, 2008. 216(2): p. 132--139.

8. Lambermont, B., et al., Large-pore membrane hemofiltration increases cytokine clearance and improves right ventricular-vascular coupling during endotoxic shock in pigs. Artif Organs, 2006. 30(7): p. 560-4.

9. Baan, J., et al., Continuous measurement of left ventricular volume in animals and humans by conductance catheter. Circulation, 1984. 70: p. 812-823.

10. Dickstein, M.L., et al., Assessment of right ventricular contractile state with the conductance catheter technique in the pig. Cardiovasc Res, 1995. 29(6): p. 820-6.

11. Nichols, W.W., et al., Input impedance of the systemic circulation in man. Circ Res, 1977. 40(5): p. 451-8.

12. Maughan, W.L., et al., Effect of arterial impedance changes on the end-systolic pressure-volume relation. Circ Res, 1984. 54(5): p. 595-602.

13. Sagawa, K., et al., Cardiac contraction and the pressure-volume relationship1988, New-York: Oxford University Press.

14. Lambermont, B., et al., Analysis of endotoxin effects on the intact pulmonary circulation. Cardiovasc Res, 1999. 41(1): p. 275-81.

15. Kass, D.A. and R.P. Kelly, Ventriculo-arterial coupling: concepts, assumptions, and applications. Ann Biomed Eng, 1992. 20(1): p. 41-62.

16. Burkhoff, D. and K. Sagawa, Ventricular efficiency predicted by an analytical model. The American journal of physiology, 1986. 250(6 Pt 2): p. R1021-7.

17. Fourie, P.R., A.R. Coetzee, and C.T. Bolliger, Pulmonary artery compliance: its role in right ventricular-arterial coupling. Cardiovasc Res, 1992. 26(9): p. 839--844.

18. Lambermont, B., et al., Effects of U-46619 on pulmonary hemodynamics before and after administration of BM-573, a novel thromboxane A2 inhibitor. Arch Physiol Biochem, 2003. 111(3): p. 217-23.

19. Smith, B.W., et al., Minimal haemodynamic system model including ventricular interaction and valve dynamics. Medical Engineering $\backslash \&$ Physics, 2004. 26(2): p. 131-139.

20. Smith, B.W., et al., Simulating transient ventricular interaction using a minimal cardiovascular system model. Physiol Meas, 2006. 27(2): p. 165--179.

21. Suga, H., K. Sagawa, and A.A. Shoukas, Load independence of the instantaneous pressure-volume ratio of the canine left ventricle and effects of epinephrine and heart rate on the ratio. Circ Res, 1973. 32(3): p. 314-22.

22. Burkhoff, D., J. Alexander, Jr., and J. Schipke, Assessment of Windkessel as a model of aortic input impedance. Am J Physiol, 1988. 255(4 Pt 2): p. H742-53.

23. Hann, C., J. Chase, and G. Shaw, Efficient implementation of non-linear valve law and ventricular interaction dynamics in the minimal cardiac model. Computer Methods and Programs in Biomedicine, 2005. 80(1): p. 65-74. 
24. Hann, C., J. Chase, and G. Shaw, Integral-based identification of patient specific parameters for a minimal cardiac model. Computer Methods and Programs in Biomedicine, 2006. 81(2): p. 181-192.

25. Hann, C.E., et al., Unique parameter identification for cardiac diagnosis in critical care using minimal data sets. Comput Methods Programs Biomed, 2010.

26. Smith and B., Minimal haemodynamic system model including ventricular interaction and valve dynamics. Medical Engineering \& Physics, 2004. 26(2): p. 131-139.

27. Smith, B.W., et al., Simulation of cardiovascular system diseases by including the autonomic nervous system into a minimal model. Comput Methods Programs Biomed, 2007. 86(2): p. 153--160.

28. Chemla, D., et al., Total arterial compliance estimated by stroke volume-to-aortic pulse pressure ratio in humans. Am J Physiol, 1998. 274(2 Pt 2): p. H500--H505.

29. Zhu, Y., J. Dai, and J.K.-J. Li, Total systemic arterial compliance: evaluation of time and frequency domain methods. Bioengineering Conference, Proceedings of the 1995 IEEE 21 Annual Northeast, 1995.

30. Lankhaar, J.-W., et al., Quantification of right ventricular afterload in patients with and without pulmonary hypertension. Am J Physiol Heart Circ Physiol, 2006. 291(4): p. H1731-1737.

31. Lankhaar, J.-W., et al., Pulmonary vascular resistance and compliance stay inversely related during treatment of pulmonary hypertension. Eur Heart J, 2008. 29(13): p. 1688-1695.

32. Guyton, A.C. and J.E. Hall, Textbook of medical physiology. 10th ed2000: W.B. Saunders Company, Philadelphia.

33. Lambermont, B., et al., Effect of hemodiafiltration on pulmonary hemodynamics in endotoxic shock. Artif Organs, 2003. 27(12): p. 1128-33.

34. Lambermont, B., et al., Time domain method to identify simultaneously parameters of the windkessel model applied to the pulmonary circulation. Arch Physiol Biochem, 1998. 106(3): p. 245-52. 


\section{Appendix}

The model is defined by Equations (A1) - (A29). A full list of the model parameters and outputs are described in Table A1 and A2.

$$
\begin{aligned}
& V_{l v f}=V_{l v}-V_{s p t} \\
& V_{r v f}=V_{r v}+V_{s p t} \\
& L_{a v} \dot{Q}_{a v}=P_{l v}-P_{a o}-Q_{a v} R_{a v} \\
& L_{m t} \dot{Q}_{m t}=P_{p u}-P_{l v}-Q_{m t} R_{m t} \\
& L_{p v} \dot{Q}_{p v}=P_{r v}-P_{p a}-Q_{p v} R_{p v} \\
& L_{t c} \dot{Q}_{t c}=P_{v c}-P_{r v}-Q_{t c} R_{t c} \\
& P_{p u}=E_{p u}\left(V_{p u}-V_{d, p u}\right)+P_{t h} \\
& P_{p a}=E_{p a}\left(V_{p a}-V_{d, p a}\right)+P_{t h} \\
& P_{v c}=E_{v c}\left(V_{v c}-V_{d, v c}\right)+P_{t h} \\
& P_{a o}=E_{a o}\left(V_{a o}-V_{d, a o}\right) \\
& P_{s y s}=E_{s y s}\left(V_{s y s}-V_{d, s y s}\right) \\
& P_{c a p}=E_{\text {cap }}\left(V_{c a p}-V_{d, c a p}\right) \\
& \dot{V}_{p v}=Q_{\text {pulout }}-Q_{m t} \\
& \dot{V}_{p a}=Q_{p v}-Q_{p u l i n} \\
& \dot{V}_{v c}=Q_{v r}-Q_{t c} \\
& \dot{V}_{a o}=Q_{a v}-Q_{s y s} \\
& \dot{V}_{s y s}=Q_{s y s}-Q_{v r} \\
& \dot{V}_{\text {cap }}=Q_{\text {pulin }}-Q_{\text {pulout }} \\
& Q_{s y s}=\frac{P_{a o}-P_{s y s}}{R_{s y s}} \\
& Q_{\text {pulin }}=\frac{P_{p a}-P_{c a p}}{R_{p u l i n}} \\
& Q_{\text {pulout }}=\frac{P_{\text {cap }}-P_{p u}}{R_{\text {pulout }}} \\
& P_{l v}=P_{l v f}+P_{p e r i} \\
& P_{r v}=P_{r v f}+P_{p e r i} \\
& P_{l v f}=d r i L \cdot E_{e s, l v f} \cdot\left(V_{l v f}-V_{d, l v f}\right)+(1-d r i L) \cdot P_{0, l v f} \cdot\left(e^{\lambda_{l v f}\left(V_{l v f} V_{0, l v f}\right)}-1\right) \\
& P_{r v f}=d r i R \cdot E_{e s, r v f} \cdot\left(V_{r v f}-V_{d, r v f}\right)+(1-d r i R) \cdot P_{0, r v f} \cdot\left(e^{\lambda_{r v f}\left(V_{r y f}-V_{0, r y}\right)}-1\right) \\
& e(t) \cdot E_{e s, s p t} \cdot\left(V_{s p t}-V_{d, s p t}\right)+\left(1-e(t) \cdot P_{0, s p t} \cdot\left(e^{\lambda_{s p p}\left(V_{s p p}-V_{0, s p t}\right)}-1\right)=\right. \\
& e(t) \cdot E_{e s, l v f} \cdot\left(V_{l v}-V_{s p t}\right)+(1-e(t)) \cdot P_{0, l v f} \cdot\left(e^{\lambda_{l v}\left(V_{l v}-V_{s p t}\right)}-1\right)-e(t) \cdot E_{e s, r v f} \cdot\left(V_{r v}+V_{s p t}\right) \\
& -(1-e(t)) \cdot P_{0, r v f} \cdot\left(e^{\lambda_{r v f}\left(V_{r v}+V_{s p t}\right)}-1\right) \\
& V_{p c d}=V_{l v}+V_{r v}
\end{aligned}
$$




$$
\begin{gathered}
P_{p c d}=P_{0, p c d}\left(e^{\lambda_{p c d}\left(V_{p c d}-V_{0, p c d}\right)}-1\right) \\
P_{p e r i}=P_{p c d}+P_{t h}
\end{gathered}
$$


Table A1: CVS model parameters

\begin{tabular}{|c|c|}
\hline Symbol & Description \\
\hline$E_{e s, l v}$ & Left ventricle end systolic elastance \\
\hline$E_{a o}$ & Aortic elastance \\
\hline$E_{v c}$ & Vena cava elastance \\
\hline$E_{e s, r v f}$ & Right ventricle end systolic elastance \\
\hline$E_{p a}$ & Pulmonary artery elastance \\
\hline$E_{p u}$ & Pulmonary vein elastance \\
\hline$E_{s y s}$ & Systemic veins elastance \\
\hline$E_{c a p}$ & Pulmonary capillaries elastance \\
\hline$R_{m t}$ & Mitral valve resistance \\
\hline$R_{a v}$ & Aortic valve resistance \\
\hline$R_{s y s}$ & Systemic vascular resistance \\
\hline$R_{t c}$ & Tricuspid resistance \\
\hline$R_{p v}$ & Pulmonary valve resistance \\
\hline$R_{\text {pulin }}$ & Pulmonary inflow resistance \\
\hline$R_{\text {pulout }}$ & Pulmonary outflow resistance \\
\hline$P_{v c}$ & Vena cava pressure \\
\hline$P_{p u}$ & Pulmonary vein pressure \\
\hline driL & Left ventricle normalised time varying elastance \\
\hline driR & Right ventricle normalised time varying elastance \\
\hline$V_{d, a o}$ & Aortic unstressed volume \\
\hline$V_{d, v c}$ & Vena cava unstressed volume \\
\hline$V_{d, p a}$ & Pulmonary artery unstressed volume \\
\hline$V_{d, p u}$ & Pulmonary vein unstressed volume \\
\hline$V_{d, s y s}$ & Systemic veins unstressed volume \\
\hline$V_{d, c a p}$ & Pulmonary capillaries unstressed volume \\
\hline$L_{m t}$ & Mitral valve inertance \\
\hline$L_{a v}$ & Aortic valve inertance \\
\hline$L_{t c}$ & Tricuspid valve inertance \\
\hline$L_{p v}$ & Pulmonary valve inertance \\
\hline
\end{tabular}


Table A2: Outputs of the CVS model

\begin{tabular}{|l|l|}
\hline Symbol & Description \\
\hline$V_{l v}$ & Left ventricle volume \\
\hline$V_{a o}$ & Aorta volume \\
\hline$V_{v c}$ & Vena cava volume \\
\hline$V_{r v}$ & Right ventricle volume \\
\hline$V_{p a}$ & Pulmonary artery volume \\
\hline$V_{p u}$ & Pulmonary vein volume \\
\hline$V_{s y s}$ & Systemic veins volume \\
\hline$V_{c a p}$ & Pulmonary capillaries volume \\
\hline$P_{l v}$ & Left ventricle pressure \\
\hline$P_{a o}$ & Aorta pressure \\
\hline$P_{v c}$ & Vena cava pressure \\
\hline$P_{r v}$ & Right ventricle pressure \\
\hline$P_{p a}$ & Pulmonary artery pressure \\
\hline$P_{p u}$ & Pulmonary vein pressure \\
\hline$P_{s y s}$ & Systemic veins pressure \\
\hline$P_{c a p}$ & Pulmonary capillaries pressure \\
\hline$Q_{m t}$ & Mitral valve flow rate \\
\hline$Q_{a v}$ & Aortic valve flow rate \\
\hline$Q_{v r}$ & Venous return flow rate \\
\hline$Q_{t c}$ & Tricuspid valve flow rate \\
\hline$Q_{p v}$ & Pulmonary valve flow rate \\
\hline$Q_{p u l i n}$ & Pulmonary inflow rate \\
\hline$Q_{p u l o u t}$ & Pulmonary outflow rate \\
\hline$V_{s p t}$ & Septum volume \\
\hline$P_{p c d}$ & Pericardium pressure \\
\hline & \\
\hline
\end{tabular}

